\title{
Neuroproliferation in the mucosa is a feature of coeliac disease and Crohn's disease
}

\author{
N Leonard, D O'B Hourihane, A Whelan
}

\begin{abstract}
The pathogenesis of villous damage in coeliac disease is unknown. Change to the delicate neuromuscular core may be significant and this study stained various categories of coeliac disease and controls with neuron-specific enolase (NSE) to examine neurofilaments in the mucosa. The amount of NSE staining was evaluated using computer image analysis. The first part of the study compared coeliac disease with Crohn's disease, carcinoma, and biopsy specimens from normal subjects. There was increased NSE staining in both the coeliac disease and Crohn's disease cases but not in carcinomas or normal controls. This difference was statistically significant. The average value for the coeliac disease patients was $\mathbf{5 0 \%}$ higher than that of Crohn's disease patients. The second part of the study compared treated coeliac disease with untreated coeliac disease. Treated coeliac disease cases had normal amounts of NSE staining, which were the same as normal controls. These findings suggest that neuroproliferation is a feature of coeliac disease and Crohn's disease. Both share a common feature - namely chronic inflammation - which has been occasionally associated with neuroproliferation. The fact that neuroproliferation resolves with treatment is further evidence for its association with chronic inflammation. The extra neuroproliferation seen in coeliac disease compared with Crohn's disease may contribute to the architectural abnormalities seen in coeliac disease.

(Gut 1995; 37: 763-765)
\end{abstract}

Keywords: Crohn's disease, coeliac disease, neuroproliferation.

While many immunological abnormalities have been recognised in coeliac disease, the primary histopathological lesion is villous atrophy with crypt hyperplasia. ${ }^{1}$ However, the specific pathological mechanisms that lead to villous damage are not known. The villus has a delicate core containing neurovascular and neuromuscular filaments, which may have a role in maintaining a normal villous architecture. Increased neurofilament has been seen in the mucosa in coeliac disease ${ }^{2}$ but has not been quantified. In an effort to study this feature more clearly we stained various coeliac disease categories and controls with an immunoperoxidase stain for neurofilaments and measured the difference between them. The control groups included patients with Crohn's disease, carcinoma, and normal biopsy specimens from endoscopic examination.

\section{Methods}

The cases were selected from the files of the Central Pathology Laboratory, St James's Hospital. Biopsy specimens were used in cases of coeliac disease (10) and normal controls (16). The cases of coeliac disease all had complete villous atrophy with a considerable increase in intraepithelial lymphocytes. Surgical resection specimens were used for cases of Crohn's disease (13) and carcinoma of the duodenum (8). The cases of Crohn's disease were from surgical resection specimens of the small intestine usually removed for intestinal obstruction. All were unequivocal cases of Crohn's disease with granulomas identified in part of the specimen. In the Crohn's disease cases areas that contained active disease were used, however, areas of ulceration were excluded. In the carcinoma cases areas immediately adjacent to the tumour were selected for analysis. In both these diseases these areas were selected so that muscularis mucosa and lamina propria could be easily identified.

Initially, several different immunoperoxidase stains were used-S100 protein, neurofilament, PGP9.5, actin, and neurone-specific enolase (NSE). NSE was found to specifically stain filamentous structures when compared with S100, neurofilament, and PGP9.5. When compared with the actin stain, the filamentous structures stained by the NSE did not stain for actin, proving that they were not of muscular origin. A monoclonal mouse antibody to NSE (Biogenex) was used here. It reacts with human gamma-gamma enolase, which is present primarily in neurons, in normal and in neoplastic neuroendocrine cells. ${ }^{3}$ It can also be expressed to some extent by platelets, megakaryocytes, $\mathrm{T}$ cells, and striated and smooth muscle cells. Platelets or megakaryocytes were not seen in the mucosa, moreover, filamentous structures were present in our slides not granular/cellular material. The possibility that the structures measured might be muscular in origin was ruled out by using the actin stain as a positive control for muscle fibres. All specimens were formalin fixed and paraffin wax embedded. Sections were cut at 4 microns thickness and stained with an antibody for NSE using a standard indirect biotinstreptavidin immunoperoxidase method. Briefly, sections were dewaxed and rehydrated through xylene and graded alcohols. Endogenous peroxidase was blocked with 
methanol/ $\mathrm{H}_{2} \mathrm{O}_{2}$. Non-specific binding was blocked with $0 \cdot 1 \%$ bovine serum albumin. Sections were incubated with appropriate primary antiserum (one hour at room temperature), followed by biotinylated rabbit-antimouse (1/300 dilution) (Dakopats) and then streptavidin-peroxidase (1/400 dilution) (Dakopats). The chromogen used was $\mathrm{DAB} / \mathrm{H}_{2} \mathrm{O}_{2}$, and sections were counterstained with haematoxylin. A negative control was used for each slide.

The amount of staining present at $\times 25$ magnification in an area of the mucosa immediately above the muscularis mucosa was evaluated using the Quantimet 570 image analyser. This image analyser can select out areas of brown immunoperoxidase staining and evaluate the area of the field covered by the stain. This value is expressed as a fraction of the field. This area was chosen because it does not contain villi and so that its shape would not change according to whether villi were present or not. Four fields were evaluated in each case in a blind fashion.

A second arm of the study aimed to see if there was a difference between treated and untreated coeliac disease patients. Cases were selected by reviewing the histological appearance. Patients were allocated to the group of treated coeliac disease patients only if their duodenal mucosa was absolutely normal with no increase in intraepithelial lymphocytes. Group 1 contained treated coeliac disease cases ( 3 patients), group 2 contained untreated coeliac cases (13 patients), group 3 contained peptic duodenitis cases (11 cases), and group 4 had normal controls (9 patients). In addition, the diameter of the nerves was measured to see if they were hypertrophic rather than hyperplastic.

Statistical evaluation used non-parametric tests as the results were not normally distributed. The Mann-Whitney test was used for the first set of results and the Wilcoxon-KruskalWallis test for the second set of results as the numbers were small.

\section{Results}

Figure 1 shows a flattened duodenal mucosa from a patient with coeliac disease that has been stained with NSE. There are prominent nerve filaments throughout the mucosa. Figure 2 shows the results of the first part of the study.

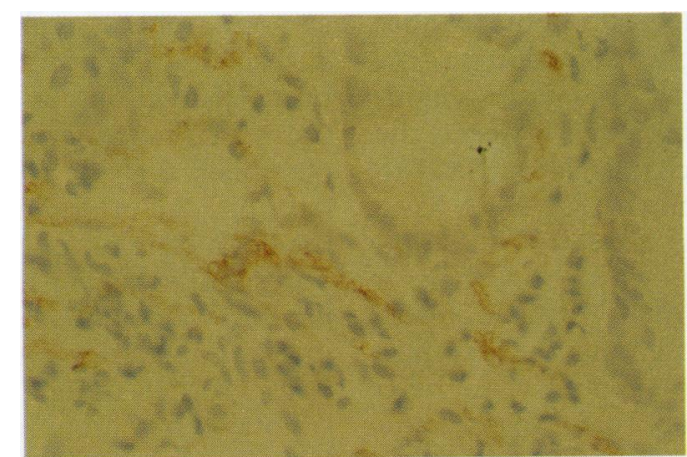

Figure 1: Immunoperoxidase stain for NSE in duodenal mucosa from a patient with coeliac disease. Neurofilaments are prominent in the mucosa between the crypts.
The average result for each group is as follows: normal controls $=0.0055$; coeliac disease $=0.0323$; carcinoma $=0.0052$; Crohn's disease $=0.0218$. The difference between normal controls and coeliac disease and between normal controls and Crohn's disease is statistically significant. (Mann-Whitney test, $\mathrm{p}<0.05$, for both comparisons.)

Figure 3 gives the results from the comparison of treated and untreated coeliac patients. The mean of the treated coeliac group was 0.036 ; of the untreated coeliac group 0.0823 ; of the duodenitis group was 0.011 , and of controls was 0.014 . These showed that there was a statistically significant difference between the group of untreated coeliac disease patients and normal controls (Wilcoxon/ Kruskal-Wallis tests, rank sum). The difference between untreated coeliac disease patients and the peptic duodenitis group was just outside statistical significance. The mean diameter of the nerve fibres in each group was as follows: normal controls $-2 \cdot 2$, untreated coeliac disease $-2 \cdot 52$, treated coeliac disease $2 \cdot 3$, and peptic duodenitis cases $-2 \cdot 34$. The

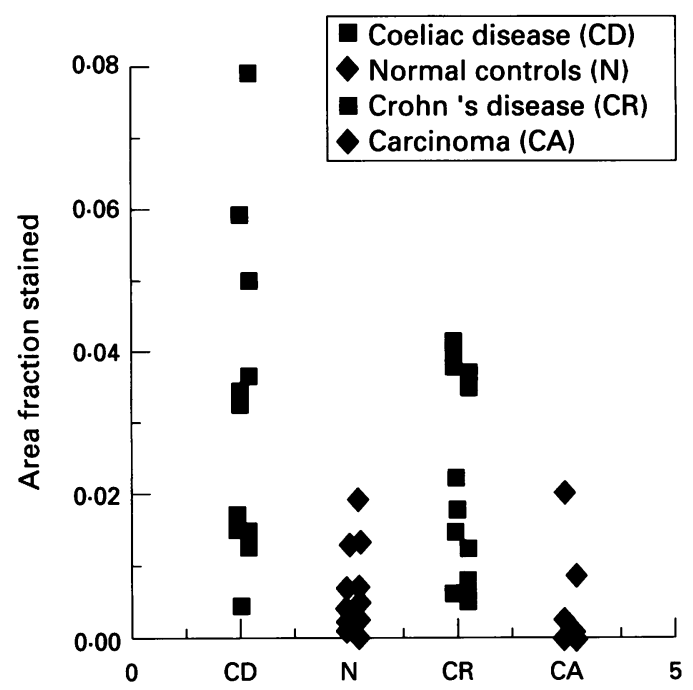

Figure 2: NSE staining in small intestinal mucosa.
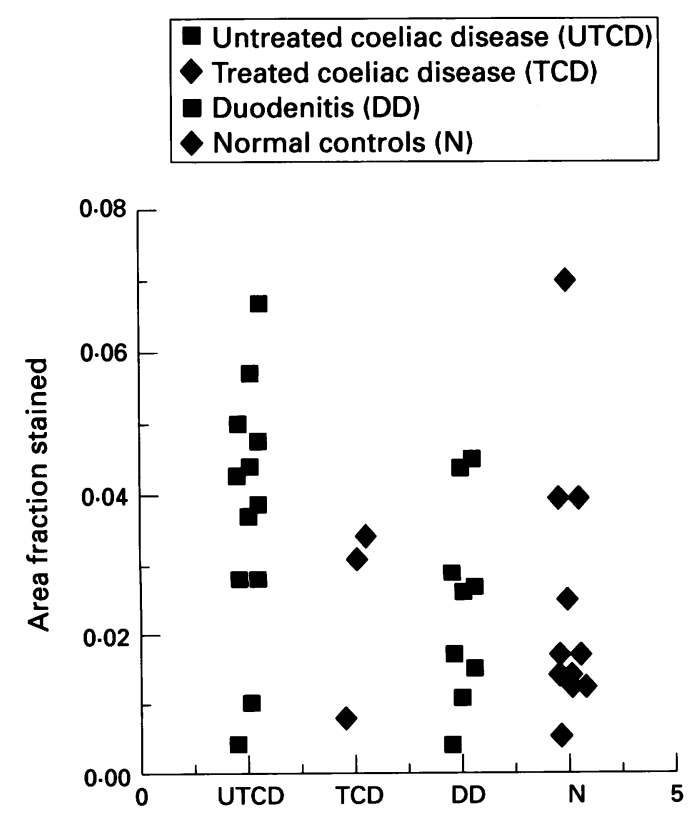

Figure 3: NSE staining in treated and untreated coeliac disease patients. 
differences between the groups was not statistically significant using the Student's $t$ test.

\section{Discussion}

The results in this study show that there is increased staining of NSE in the mucosa in both coeliac disease and Crohn's disease. While hypertrophic nerves have been seen in the submucosa in colon with Crohn's disease, the presence of increased nerve filaments in the mucosa in coeliac disease and Crohn's disease has not been reported before. It is noteworthy that the hypertrophic nerves seen in Crohn's disease are very different from the phenomen reported here. In Crohn's disease there are abnormally large and irregularly shaped nerve trunks in various bowel layers but sparing the mucosa, a very different finding from the proliferation of small nerve filaments described here in the mucosa. ${ }^{4} \mathrm{~A}$ common feature in both of these diseases is the presence of chronic inflammation. Neuroproliferation is not a feature that is commonly commented on in chronic inflammation and there are only two reports of its involvement in chronic inflammation - in the proctocolitis associated with lymphogranuloma venereum ${ }^{5}$ and also in the obliterated appendix, which is thought to be a result of repeated minimal subclinical attacks of inflammation. ${ }^{6}$ The finding here that neurofilaments return to normal in fully treated coeliac disease, which not only has normal architecture but also no increase in inflammatory cells, points to a cause and effect relation.

During the course of chronic inflammation there is a potential for the release of neurotoxins. Mast cells and eosinophils, in particular, contain several neurotoxins such as major basic protein, eosinophil specific cationic protein, eosinophil derived neurotoxin, and eosinophil peroxidase. ${ }^{7}$ Previous papers have noted that eosinophils and mast cells are increased in coeliac disease..$^{8-11}$ One interpretation of these findings is that neural damage from neurotoxins released during chronic inflammation may lead to a compensatory neuroproliferation. What this study is unable to show is whether overall neural activity is increased, decreased or remains the same.

The cause of the architectural abnormalities in coeliac disease is unknown. The centre of the villus is made up of a delicate neurovascular/ muscular core. Changes in neural interactions in the mucosa could bring about a change in villus growth and maintenance of a normal structure. When the values are reviewed, the value for coeliac disease is 0.0323 versus 0.0218 for Crohn's disease. Although the difference does not reach statistical significance the value for coeliac disease is $50 \%$ larger than that for Crohn's disease. It might be postulated that this extra amount of neuroproliferation is necessary to produce the architectural abnormalities seen in coeliac disease, which is not a feature of Crohn's disease.

In conclusion, NSE staining is significantly increased in the mucosa of patients with coeliac disease and Crohn's disease, a feature that has not been reported before. This change is more noticeable in coeliac disease than in Crohn's disease. This increase seems to be associated with chronic inflammation as it returns to normal once coeliac disease is treated. The presence of neuroproliferation in both of these diseases offers new approaches to their study as their causes and pathogenesis are as yet incompletely understood.

1 Trier JS. Coeliac sprue, review article. $N$ Engl F Med 1991; 325: $1709-18$

2 Whelan A, Williams Y, Lydyard P, O'Keefe J, Kelleher D, Weir DG. The inflammatory response in the small intestine of patients with coeliac disease. In: Feighery C, sensitive disease. Dublin: Oak Tree Press, 1994: 253-63.

3 Biogenex data sheet on monoclonal antibody to neuron specific enolase. Upton upon Severn, Worcestershire: Biogenix Diagnostics.

4 Dvorak AM, Osage JE, Monahan RA, Dickerson RD. Crohn's disease: transmission electron microscopic studies. III Target Tissues. Proliferation of an injury to smooth muscle and the autonomic nervous system. Hum Pathol 1980; 11: 620-34.

5 De La Monte S, Hutchins $M$. Follicular proctocolitis and neuromatous hyperplasia with lymphogranuloma venereum. Hum Pathol 1985; 16: 1025-32.

6 Olsen BS, Holck S. Neurogenous hyperplasia leading to appendiceal obliteration: an immunohistochemical study of 237 cases. Histopathology 1987; 11: 843-9.

7 Hallgren R, Colombel JF, Dahl R, Fredens K, Kruse A, Jacobsen ND, et al. Neutrophil and eosinophil involvement of the small bowel in patients with coeliac disease and Crohn's disease: studies on the secretion rate and immunohistochemical localisation of granulocyte granule constituents. Am $\mathcal{F}$ Med 1989; 86: 56-64.

8 Lavo B, Kuntson L, Loof L, Odlind B, Venge P, Hallgren R. Am f Med 1989; 87: 655-60.

9 Talley NJ, Kephart GM, McGovern TW, Carpenter HA Gleich GJ. Deposition of eosinophil granule major basic protein in eosinophilic gastroenteritis and coeliac disease Gastroenterology 1992; 103: 137-45.

10 Loft DE, March MN, Sandle GI, Crowe PT, Garner V, Gordon $\mathrm{D}$, et al. Studies of intestinal lymphoid tissue. XII. Epithelial lymphocyte and mucosal responses to rectal gluten challenge in coeliac sprue. Gastroenterology rectal gluten chal

11 Strobel S, Busuttil A, Ferguson A. Human intestinal mucosal mast cells: expanded population in untreated coeliac disease. Gut 1983; 24: 222-7. 